\title{
Design investigation of electromechanical generator for energy harvesting
}

\author{
Nejc Smolar ${ }^{1}$, and Peter Virtič ${ }^{1, *}$ \\ ${ }^{1}$ University of Maribor, Faculty of Energy Technology, Hočevarjev trg 1, 8270, Krško, Slovenia
}

\begin{abstract}
In this paper designs of electromechanical generator for low frequency energy harvesting have been investigated. Simulation with finite element method has been conducted in order to determine highest output voltage of simple and robust generator consisting of permanent magnet and windings. In first part round magnets have been used in spherical and cylindrical form, benefiting from their ability to roll through winding with almost no mechanical friction inducing voltage in into windings. In the second part spindles with smaller radius than circumference of magnet were added to axis to increase rotational velocity of magnet in ambition to further increase induced voltage. As a result of added spindles and use of different magnet shapes length of winding turn varied and resistance of winding varied with it. To ensure similar conditions, windings have been recalculated to lowest electrical resistance using same fill factor, resulting in less winding turns decreasing induced voltage. In case of same kinetic energy input, higher rotational velocity combined with lower inertia produced higher induced voltage output.
\end{abstract}

\section{Introduction}

Constant power supply offshore always presents challenge, especially for self-sustaining applications. Where during the night most of energy is consumed powering lights to ensure night visibility, for maritime safety. Also considering that sizable amount of vessels are anchored in natural bays used only occasionally or for few months a year, but they still need to retain minimum electricity supply for mast light and to ensure that starter battery does not fully discharge.

Hence, durable self-sustaining systems are fundamental for such applications. Since PV panels are one of more popular choices for self-sustaining systems, due to abundance of solar energy, such systems are not always optimal choice. Especially as solar powered systems have big disadvantage in cloudy/foggy conditions and at high latitudes, where daily solar energy over winters is very low.

Great deal of research has been done on providing power for self-sustainable systems. Wave energy harvesting sea buoys are one of them, although main focus is on providing energy for seabed anchored buoys, where linear generators are mostly used [1-4] offering

\footnotetext{
* Corresponding author: peter.virtic@um.si
} 
high energy density. However harvesters as such are not feasible for moving objects, thus some research have been done for non-stationary applications [5-12].

In prospect to create simple, durable and cost efficient design of generator that can generate sufficient electrical energy on the move, design investigation with simulations, has been conducted. This paper proposes electromagnetic generator with varying geometries of magnets and windings, to determine combination with highest output voltage.

\section{Design investigation with FEM analysis}

\subsection{Principle of operation}

Generator design consists of compact windings aligned with $\mathrm{XY}$ plane and two-pole $\mathrm{NdFeB}$ permanent magnet (PM), as presented Fig. 1 shows, which is in first case spherical and in second case cylindrical shape. Magnets magnetization orientation is perpendicular to the PM axis plane.

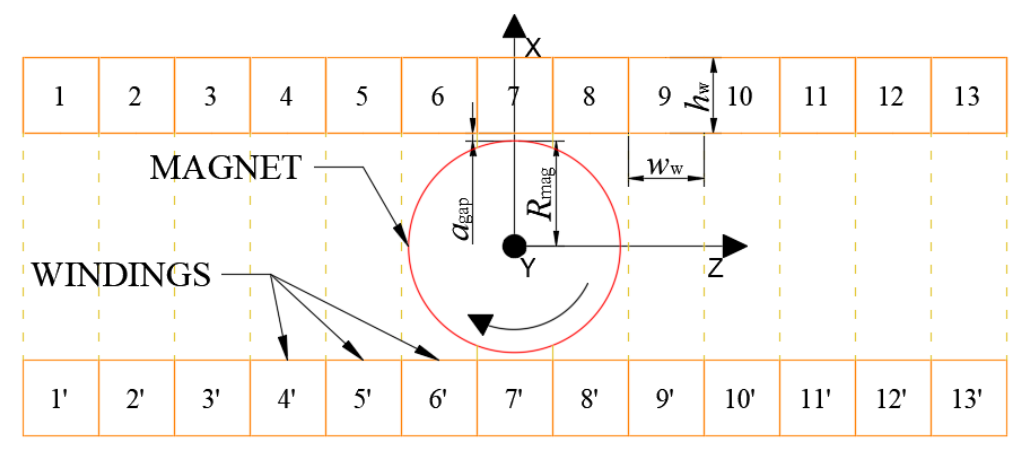

Fig. 1. XZ cross section of model.

Idea is that when the generator is subjected to the alternating inclination change, PM inside of device starts to roll through winding along $\mathrm{Z}$ axis. Thus inducing voltage into surrounding winding corresponding to Lenz's law.

Induced voltage waveform there by varies as result of magnetic flux time derivative, which depends on number of factors. Firstly on proximity of PM to observing winding, due to exponential weakening of magnetic field intensity with the distance. Secondly, depending on magnet's magnetization angle at given point compared to the winding's plane normal ( $\mathrm{Z}$ axis), as it was already discussed for the spherical magnet in [9]. Thirdly, depending on the winding geometry and number of turns. And lastly, depending also on flux leakage in air gap and to the sides of winding.

\subsection{Scope of investigation}

Shape of magnets was limited to sphere and three dimensions of cylinder, as presented in Fig. 2, where spheres radius $R_{\mathrm{S}}$ of $20 \mathrm{~mm}$ determined volume of PM for cylinder shapes. Cylinder radii wearied from 14 to $22 \mathrm{~mm}$, where cylinder radius $R_{\mathrm{C} 22}$ of $22 \mathrm{~mm}$ was presenting shortest wire loop around $\mathrm{PM}$, cylinder with radius $R_{\mathrm{C} 17.5}$ of $17.5 \mathrm{~mm}$ was presenting square axis intersection and $R_{\mathrm{C} 14}$ was presenting cylinder PM with double height compared to diameter. 
In first case, PM with dimensions stated in previous paragraph, were assumed to roll on their own base/circumference trough the windings. In second case spindles were added to magnets as shown on lower part of Fig. 2. Hence reducing rolling circumference to radius $r$ of $2 \mathrm{~mm}$, similarly as previously done in [9], forcing magnet to higher angular velocity and making less displacement along $\mathrm{Z}$ axis in one rotation.

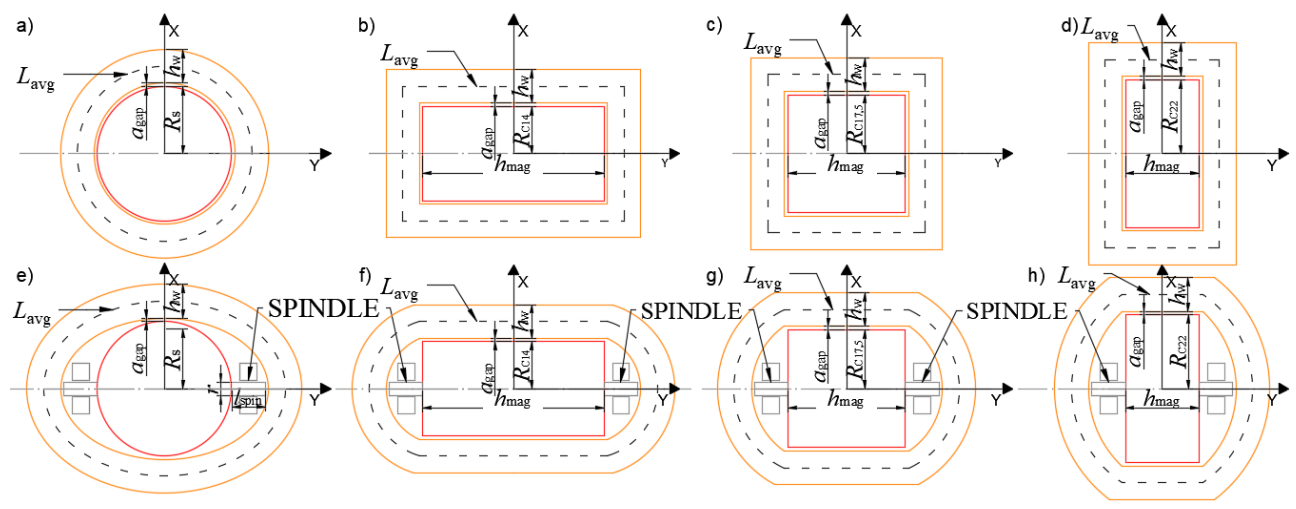

Fig. 2. XY plane cross sections of a) sphere, b) cylinder with radius of $14 \mathrm{~mm}$, c) cylinder with radius of $17.5 \mathrm{~mm}, \mathrm{~d}$ ) cylinder with radius of $22 \mathrm{~mm}$, e) sphere with spindle, f) cylinder with radius of $14 \mathrm{~mm}$ with spindle, $\mathrm{g}$ ) cylinder with radius of $17.5 \mathrm{~mm}$ with spindle, h) cylinder with radius of $22 \mathrm{~mm}$ with spindle.

To establish comparable design conditions height $h_{\mathrm{w}}$ and width $w_{\mathrm{w}}(10$ by $10 \mathrm{~mm})$ of windings staid the same and contained 100 turns of wire $(N)$. Presuming filling factor of 0.71 (100 turns with nominal diameter of $0,95 \mathrm{~mm}$ wire). Air gap $a_{\text {gap }}$ between magnet and winding was equal to $1.5 \mathrm{~mm}$.

\subsection{Modelling}

Due to the need for simplification we assumed that spherical PM magnetization vector was always aligned with $\mathrm{XZ}$ plane, which is not fully true for sphere without spindles, where orientation of magnetic field can deviate from alignment with $\mathrm{XZ}$ plane. With this presumption we ensure that magnetic field cut winding at right angle, thus generating maximum induced voltage.

Magnet's velocity $v$ along $\mathrm{Z}$ axis was constant and equal to the velocity of rolling object after $0.5 \mathrm{~m}$ of height difference $\mathrm{H}$, presented in equation (1), where $m$ presents mass of magnet and $g$ gravitational acceleration. Velocity of magnet varied correspondingly to magnets inertia $I$ and rolling base radius $r_{\text {base, }}$, which was in case of no spindles determined with PM radius and in case of spindles with radius of spindle.

$$
v=\sqrt{\frac{2 m g H}{m+\frac{I}{r_{\text {base }}^{2}}}}
$$

Sphere's velocity is slightly higher than cylinder's $(2.65 \mathrm{~m} / \mathrm{s}$ compared to $2.56 \mathrm{~m} / \mathrm{s})$, if rolling on their circumference. On the other hand with equal radius of spindles, velocity along $\mathrm{Z}$ axis is determined only by magnets inertia. 
With previously stated conditions 3D magnetostatic model was created in ANSYS Maxwell using finite element method. Simulations output was winding's magnetic flux, which was then transformed to induced voltage using the ratio of flux difference and time difference between two sequential calculated points.

\subsection{Effect of design on induced voltage and resistance}

\subsubsection{Models without spindles}

As specified in section 2.2 we firstly simulated magnets rolling on their circumference, as suggested in upper half of Fig. 2, assuming their equal conditions of operation. Rotational motion and displacement along $Z$ axis created periodical waveform, presented in Fig. 3 . Where observed winding's induced voltage peak was generated when magnet was closest to it, taking in to consideration magnetic field alignment with $\mathrm{Z}$ axis.

Symmetry of induced voltages occurs only in case of magnet's field alignment with $\mathrm{Z}$ axis and at the same instance being in exact centre of winding. Hence producing equal positive and negative peaks and therefore maximum peak to peak induced voltage of one winding.

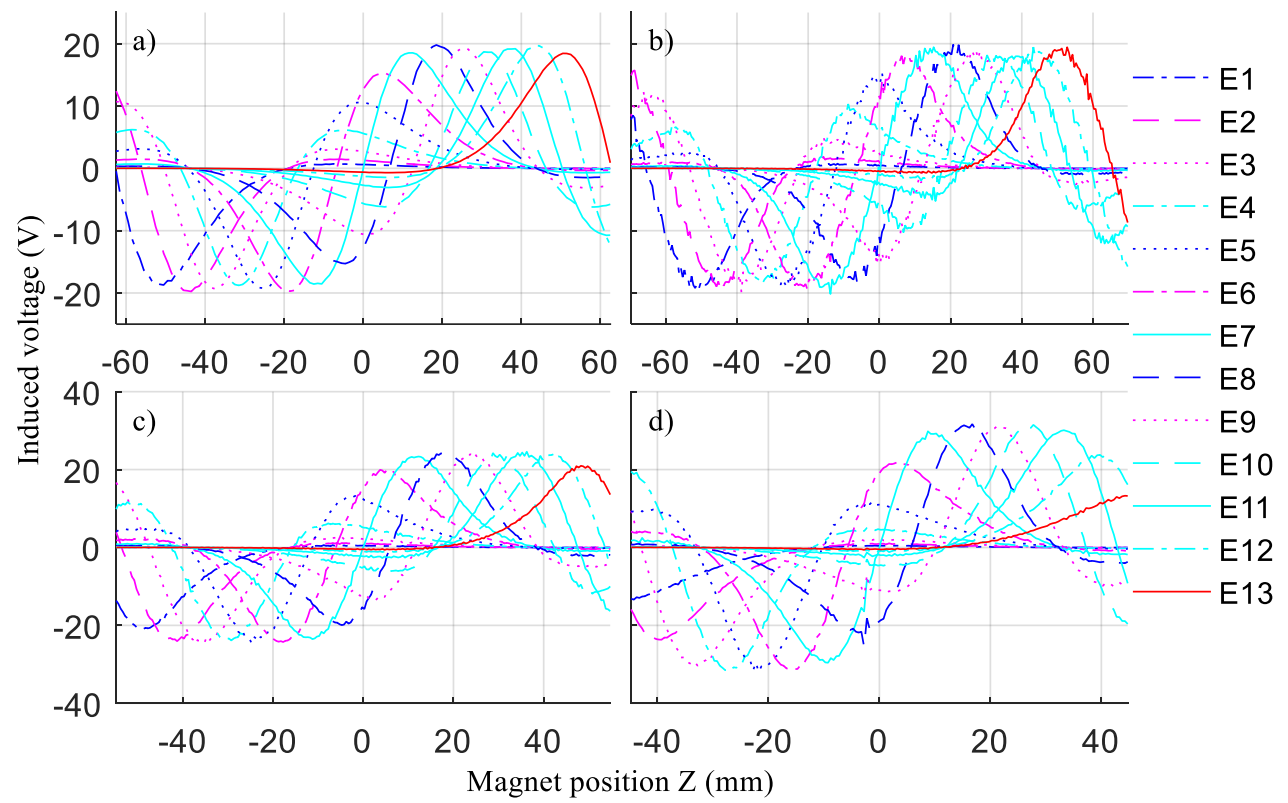

Fig. 3. Induced voltage waveform of 13 windings for magnets rolling on circumference: a) sphere, b) cylinder with radius of $22 \mathrm{~mm}$, c) cylinder with radius of $17.5 \mathrm{~mm}$, d) cylinder with radius of $14 \mathrm{~mm}$.

Closely comparing symmetrical induced voltages in Fig. 4, for all four designs, the highest winding output can be determined. As shown in Fig. 4 highest peak to peak value of $59,42 \mathrm{~V}$ was achieved by cylinder with radius of $14 \mathrm{~mm}$, mainly due to higher rotational velocity and consequently flux change rate.

Second highest peak to peak value of $46.88 \mathrm{~V}$ was produced by cylindrical magnet square cross section. Cylinder with smallest loop around axial cross section and spherical magnets generated lowest induced voltages of 39.6 and $37.09 \mathrm{~V}$. 


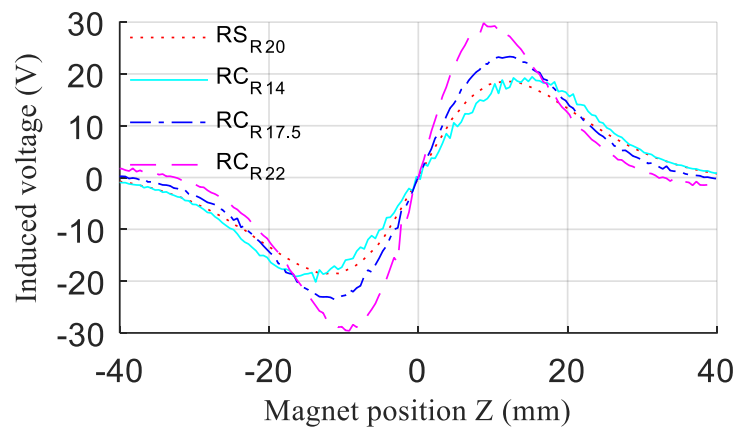

Fig. 4. Comparison of induced voltage for sphere and cylinders with radii from 14 to $22 \mathrm{~mm}$ rolling on circumference.

\subsubsection{Models with spindle}

To increase flux change rate, spindles with radius of $2 \mathrm{~mm}$ (Fig. 2) were added to magnet's axis to force magnet in turning with higher angular velocity, with smaller displacement in $\mathrm{Z}$ axis, therefore peak to peak induced voltages increased and were more in phase as seen in Fig. 5, compared to values without spindle (Fig. 3), where peaks of induced voltage were more evenly distributed.

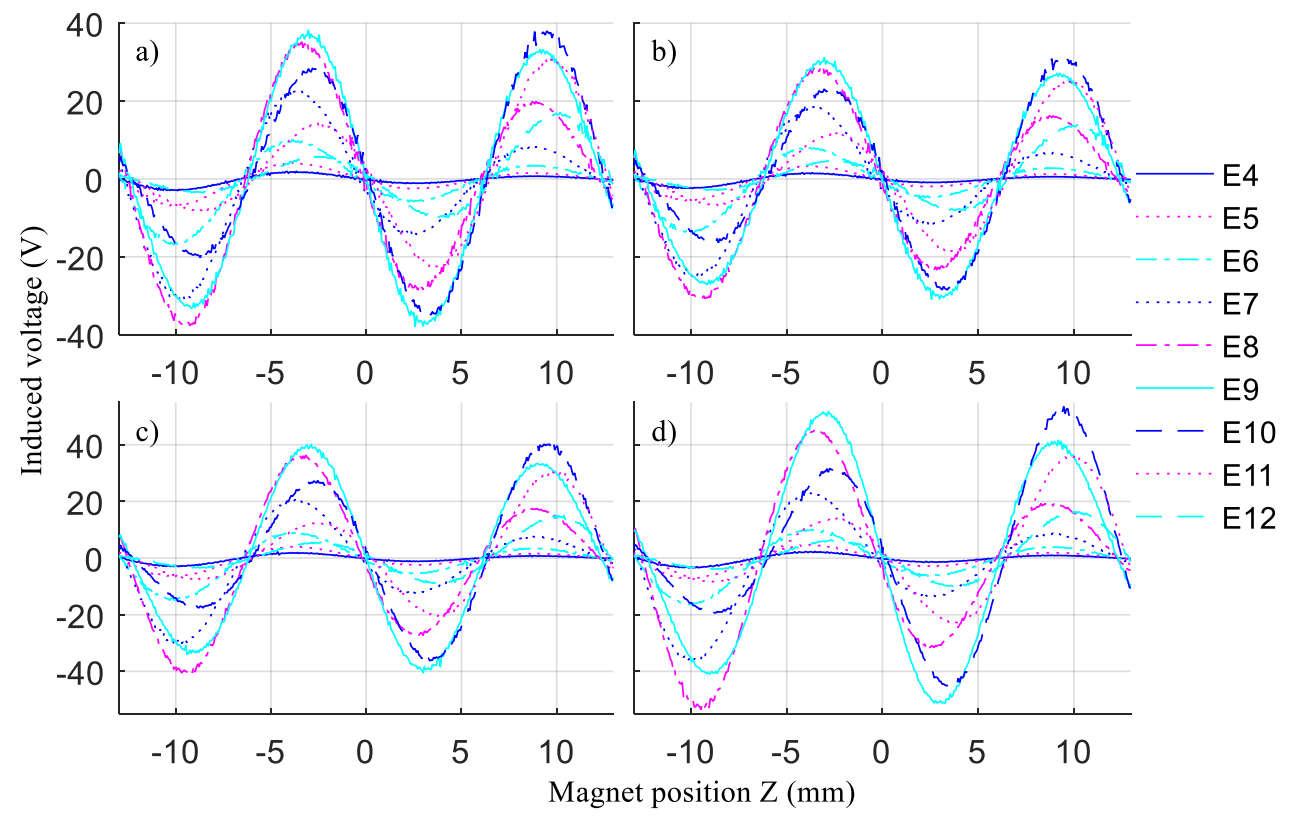

Fig. 5. Induced voltage waveform of 9 windings for magnets rolling on $2 \mathrm{~mm}$ spindle: a) sphere, b) cylinder with radius of $22 \mathrm{~mm}$, c) cylinder with radius of $17.5 \mathrm{~mm}$, d) cylinder with radius of $14 \mathrm{~mm}$.

Biggest improvement was noticeable on sphere shape, where peak to peak voltage almost doubled and reached 73.26 V. Although highest peak to peak induced voltage of one 
winding was generated with $14 \mathrm{~mm}$ radius of cylinder with spindles $(98.81 \mathrm{~V})$ as seen in Fig. 6.

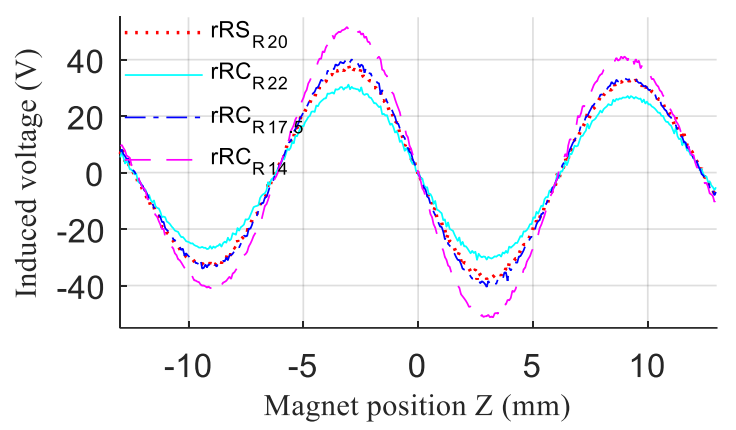

Fig. 6. Comparison of induced voltage for sphere and cylinders with radii from 14 to $22 \mathrm{~mm}$ rolling on spindle.

As consequence of using different shapes of magnets and added spindles, length of winding changed, as shown on Fig. 2, where average length of turn was presented with dashed line. Therefore with usage of same fill factor resistance changed as well. Total length of each winding was derived from average length of turn and written in equations (2-5). Where $R_{\text {mag }}$ and $h_{\text {mag }}$ stand for radius and height of magnet and $l_{\text {spin }}$ is the length of spindle.

$$
\begin{aligned}
& L_{\mathrm{RS}}=N 2 \pi\left(R_{\mathrm{mag}}+a_{\mathrm{gap}}+w_{\mathrm{w}}\right) \\
& L_{\mathrm{RC}}=4 N\left(R_{\mathrm{mag}}+2 a_{\mathrm{gap}}+w_{\mathrm{w}}+\frac{h_{\mathrm{mag}}}{2}\right) \\
& L_{\mathrm{rRS}}=N 2 \pi \sqrt{\frac{\left(R_{\mathrm{mag}}+a_{\text {gap }}+\frac{w_{\mathrm{w}}}{2}\right)^{2}+\left(\frac{h_{\mathrm{mag}}}{2}+l_{\text {spin }}+a_{\mathrm{gap}}+\frac{w_{\mathrm{w}}}{2}\right)^{2}}{2}} \\
& L_{\mathrm{rRC}}=4 N\left(\frac{h_{\text {mag }}}{2}+\frac{\left(l_{\text {spin }}+a_{\mathrm{gap}}+\frac{w_{\mathrm{w}}}{2}\right)^{2}+\left(\frac{R_{\mathrm{mag}}}{2}+a_{\mathrm{gap}}+\frac{w_{\mathrm{w}}}{2}\right)^{2}}{2\left(l_{\mathrm{spin}}+a_{\mathrm{gap}}+\frac{w_{\mathrm{w}}}{2}\right)} \cdot \arcsin \left(\frac{2\left(l_{\text {spin }}+a_{\mathrm{gap}}+\frac{w_{\mathrm{w}}}{2}\right) \cdot\left(\frac{R_{\text {mag }}}{2}+a_{\mathrm{gap}}+\frac{w_{\mathrm{w}}}{2}\right)}{\left(l_{\mathrm{spin}}+a_{\mathrm{gap}}+\frac{w_{\mathrm{w}}}{2}\right)^{2}+\left(\frac{R_{\text {mag }}}{2}+a_{\text {gap }}+\frac{w_{\mathrm{w}}}{2}\right)^{2}}\right)\right)
\end{aligned}
$$

Total length of wire needed for winding sphere design was $16.3 \mathrm{~m}$ and $19.8 \mathrm{~m}$ with spindles. Cylinders winding length for $14 \mathrm{~mm}$ radius was $21.2 \mathrm{~m}$ and $21.9 \mathrm{~m}$ with spindles, for $17.5 \mathrm{~mm}$ radius was $18,8 \mathrm{~m}$ and 19.2 with spindles and for shortest loop cylinder was needed $18.0 \mathrm{~m}$ of wire without spindles and $3 \mathrm{~cm}$ more with them.

\section{Design comparison and discussion}

Comparing all eight designs we can group them in two construction groups. First one without spindle and second one with them. Where sphere without spindles is not feasible design as such, due to magnetics' field tendency to align itself with axis of rotation. There 
have been already ideas how to solve this problem without need for spindles. On the other hand this design can make a good reference for comparison to other designs.

To reach highest potential difference at any given moment, every second winding has been inverted, thus resulting in opposing polarities of neighbouring windings. From potential difference (solid line) of positive (dash-dot line) and negative voltage wave curve (dot line), root mean square (RMS) voltage (dash line) was calculated and presented in Fig. 7. Where designs without spindle are presented on top half of Fig. 7 and on the bottom half are designs with spindle.

Comparing induced voltages, it is obvious that higher angular speed increased induced voltage RMS value from $23 \%$ to $53 \%$. Highest increase was observed with sphere and lowest with $22 \mathrm{~mm}$ radius cylinder.
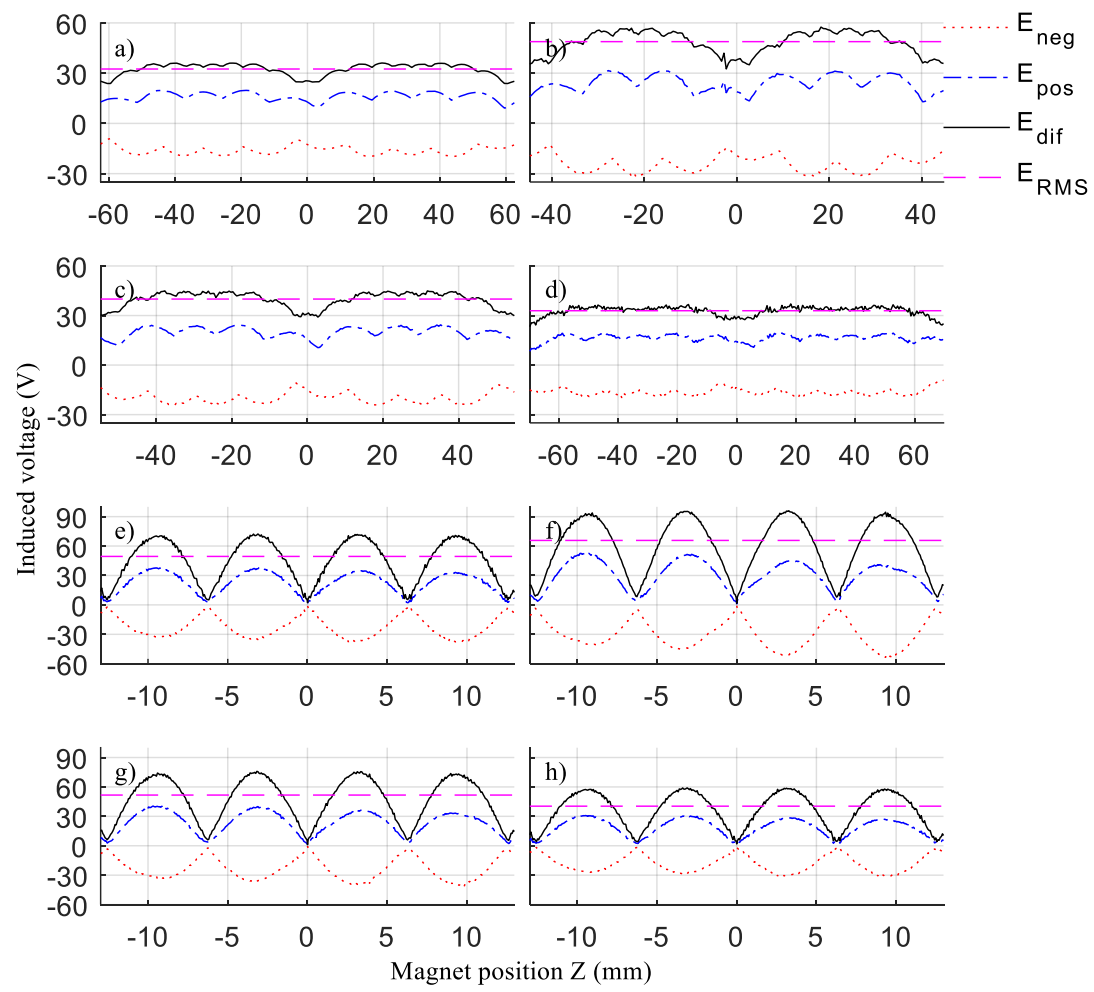

Fig. 7. Positive and negative induced voltage curve, their potential difference and RMS value.

a) sphere without spindles, b) $14 \mathrm{~mm}$ cylinder without spindles, c) $17.5 \mathrm{~mm}$ cylinder without spindles,

d) $22 \mathrm{~mm}$ cylinder without spindles, e) sphere with spindles, f) $14 \mathrm{~mm}$ cylinder with spindles,

g) $17.5 \mathrm{~mm}$ cylinder with spindles, h) $22 \mathrm{~mm}$ cylinder with spindles.

Highest voltage output designs in both cases were cylinders with radius of $14 \mathrm{~mm}$, producing $48.31 \mathrm{~V}$ without spindles and $67.51 \mathrm{~V}$ with them. Second highest voltage was achieved in both cases by cylinder with radius of $17.5 \mathrm{~mm}$, generating $39.97 \mathrm{~V}$ without spindles and $51.7 \mathrm{~V}$ with them. Lowest RMS voltages were produced with sphere and cylinder with square axis cross section. In case of cylinder without spindles $32.91 \mathrm{~V}$ was generated, $0.44 \mathrm{~V}$ more than by sphere. In other instance where spindles were used sphere produced $9.07 \mathrm{~V}$ more than $22 \mathrm{~mm}$ cylinder with $40.37 \mathrm{~V}$. Taking into consideration differences in geometry, the use of spindles increased the length of windings, due to increase in average length of turn, as shown in Fig. 2. RMS induced voltages $(E)$ were 
recalculated for the lowest resistance $R_{\mathrm{RS}}$ (winding of sphere without spindles), taking into account the same fill factor $(F F)$ of 0,71 and total length of winding $l_{\text {avg }}$, thus changing number of turns and wire area using equation (6), where $\rho_{\mathrm{Cu}}$ electrical resistivity and $A_{\mathrm{w}}$ presents cross section area of winding and equals to $h_{\mathrm{w}}$ times $w_{\mathrm{w}}$.

$$
E_{\mathrm{eqR} \mathrm{RS}_{\mathrm{RS}}}=\frac{E}{N} \sqrt{\frac{R_{\mathrm{RS}} \cdot A_{\mathrm{w}} \cdot F F}{\rho_{\mathrm{Cu}} \cdot l_{\mathrm{avg}}}}
$$

Recalculated RMS voltages $\left(E_{\text {eqRes }}\right)$ decreased, due to lower number of turns, although order of designs by $E_{\text {eqRes }}$ did not change compared to previously stated one with voltages generated at 100 turns. Highest recalculated value decrease of $9.3 \mathrm{~V}$ was experienced by cylinder with radius of $14 \mathrm{~mm}$, still having $10.5 \mathrm{~V}$ higher induced voltage compared to second highest cylinder with $17.5 \mathrm{~mm}$ radius.

\section{Conclusion}

In prospect to create feasible and simple designs of electromagnetic generator for low frequencies, eight proposed geometries were simulated and analysed. Focusing on highest voltage output at limited winding space induced voltage and internal resistance were observed. As a consequence of shape difference, velocity of rolling magnet was determined by the circumference and inertia, where both played an important role. Higher induced voltages were generated by faster rotating magnets. Therefore spindles were introduced to designs to further increase angular velocity increasing also axial length. Higher winding resistance caused additional voltage drop that had to be taken into account at power generation. Therefore recalculated RMS voltages have been introduced to ensure equal resistance. According to recalculated RMS voltages highest output was achieved by the smallest inertia design. Cylinder with four to one ratio between height and radius rolling on spindles and lowest voltages were obtained by the highest inertia cylinder without spindles.

\section{References}

1. S. Hor, A. Tabesh, A. Zamani, Proc. IET RPG (2011)

2. A. Pirisi, M. Mussetta, F. Grimaccia, D. Caputo, G. Gruosso, R. E. Zich, Proc. of $6^{\text {th }}$ IET PEMD (2012)

3. H. Li, P. Pillay, IEEE Trans. on In. App. 47, 2445-2452 (2011)

4. L. Huang, J. Liu, H. Yu, R. Qu, H. Chen, H. Fang, IEEE Trans. Appl. Supercond. 25, 5202505 (2015)

5. G. Bracco, E. Giorcelli, C. Attaianese, IEEE ECCE, 3864-3871 (2011)

6. J. A. Member, M. R. Burkhardt, S. Member, F. Davoodi, J. W. Burdick, IEEE ECCE, 3167-3171 (2015)

7. M. R. Burkhardt, F. Davoodi, J. W. Burdick, F. Davoudi, IEEE ICRA, 2665-2672 (2014)

8. S. Wu, P. C. K. Luk, C. Li, X. Zhao, Z. Jiao, IEEE Trans. Magn. 53, 4600706 (2017)

9. N. Fondevilla, C. Serre, J. Esteve, Proc. of Int. Conf. Power Eng. Energy Electr. Drives (2011)

10. J. Asama, M. R. Burkhardt, F. Davoodi, J. W. Burdick, IEEE ICRA, 244-251 (2015)

11. B. J. Bowers, D. P. Arnold, JMM 19, 094008 (2009)

12. J. Joos, O. Paul, IEEE SENS. 1 (2015) 